\title{
BER Performance Analysis of Multistage PIC Scheme in An Asynchronous DS-CDMA System with Long Scrambling Codes
}

\author{
Pei Xiao, Erik Ström \\ Communication Systems Group, Dept. of Signals and Systems \\ Chalmers University of Technology, SE-412 96, Göteborg, Sweden \\ phone: +46 317721768 , fax: +46 317721748 \\ E-mail: pei.xiao@s2.chalmers.se, erik.strom@s2.chalmers.se
}

\begin{abstract}
In this paper, we tackle the problem of theoretical evaluation for the multistage parallel interference cancellation (PIC) scheme in a DSCDMA system with orthogonal modulation and long scrambling codes. The studied system operates on the reverse link in a time-varying multipath Rayleigh fading channel. By applying the Central Limit Theorem to multiple access interference (MAI) and intersymbol interference (ISI), as well as identically distributed chips from a single interferer, the bit error rate (BER) performance of the PIC scheme at any stage can be recursively computed from the signal-to-noise ratio, number of users, the number of paths per user, processing gain of the CDMA system, as well as the average received power of each path. The proposed approximative analysis is validated by the MonteCarlo simulations and proved to be accurate, and it gives insight into the performance and capacity one can expect from the PIC based receivers under different situations.
\end{abstract}

\section{INTRODUCTION}

The system under study is an uncoded asynchronous DSCDMA system with orthogonal signaling formats. The transmitted chip sequence from a particular user is the concatenation of one of $M$ possible Walsh sequences (representing the transmitted symbol) and a long scrambling code. The Walsh (Hadamard) code is employed for combining the advantages of spreading and coding to achieve improved performance for spread spectrum (CDMA) systems.

The performance of orthogonal modulated DS-CDMA system with noncoherent and coherent combining was evaluated analytically in $[1,2]$ and in $[3]$ respectively. The performance of interference canceler for short-code CDMA systems with BPSK signaling was investigated, e.g., in [4-6]. An adaptive multistage PIC scheme was analyzed in [4], and a closed form expression for BER performance is presented for the system operating over AWGN channels. The BER expressions are extended to derive asymptotic limits on the performance of interference cancellation as the number of cancellation stages approaches infinity, demonstrating a fundamental limit on the performance that can be expected from the multistage PIC scheme. In [5], an analytical BER expression for an adaptive multistage interfer- ence canceler was presented using an improved Gaussian approximation. The inclusion of second order statistics of MAI allows better performance prediction in cases where interference power has a random distribution, and it can be used to evaluate the performance of multistage PIC in arbitrary fading environments.

However, to our best knowledge, no results on the performance analysis of PIC for long-code CDMA systems in general, and PIC for orthogonal modulated CDMA systems in particular are available in the existing literature. The previous performance evaluation only relied on the use of simulation techniques. In this paper, we provide an analytical approach to assess the performance of PIC for the system in question.

\section{System Model and Receiver Algorithms}

Fig. 1 shows the signal path for the user $k$. The $k^{t h}$ user $j^{t h}$ transmitted symbol is denoted by $i_{k}(j) \in\{1,2, \cdots, M\}$, and is mapped into $\mathbf{w}_{k}(j) \in$ $\left\{\mathbf{w}_{1}, \mathbf{w}_{2}, \cdots, \mathbf{w}_{m}, \cdots, \mathbf{w}_{M}\right\}$, which is one of the $M$ orthogonal signal alternatives. The Walsh codeword $\mathbf{w}_{k}(j) \in$ $\{+1,-1\}^{M}$, is then repetition encoded into $\mathbf{s}_{k}(j) \in$ $\{+1,-1\}^{N}$ so that each bit of the Walsh codeword is spread (repetition coded) into $N_{c}=N / M$ chips, and each Walsh symbol is represented by $N$ chips. The Walsh sequence $\mathbf{s}_{k}(j)$ is then scrambled (randomized) by a scrambling code unique to each user to form the transmitted chip sequence $\mathbf{a}_{k}(j)=\mathbf{C}_{k}(j) \mathbf{s}_{k}(j) \in\{+1,-1\}^{N}$ where $\mathbf{C}_{k}(j) \in\{-1,0,+1\}^{N \times N}$ is a diagonal matrix whose diagonal elements correspond to the scrambling code for the $k^{t h}$ user's $j^{t h}$ symbol. The purpose of scrambling is to separate users. In this paper, we focus on the use of long codes, e.g., the scrambling code differs from symbol to symbol.

The baseband signal is formed by pulse amplitude modulating $\mathbf{a}_{k}(j)$ with a unit-energy rectangular chip waveform $\psi(t)$. The baseband signal is then multiplied with a carrier and transmitted over a Rayleigh fading channel with $L_{k}$ resolvable paths, having time-varying complex channel gains $h_{k, 1}(t), h_{k, 2}(t), \ldots, h_{k, L_{k}}(t)$ and delays $\tau_{k, 1}, \tau_{k, 2}, \ldots, \tau_{k, L_{k}}$. The received signal is the sum of $K$ users' signals plus additive white complex Gaussian noise $n(t)$. The received vector corresponding to the $k^{t h}$ user's $j^{t h}$ symbol after frequency down-conversion and chip matched filtering is de- 


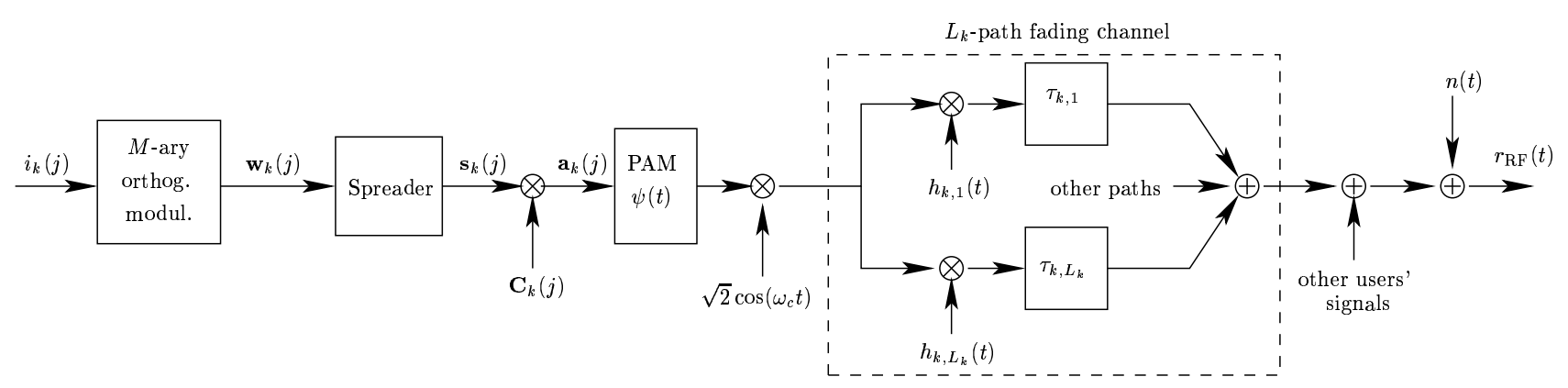

Fig. 1. Block diagram of the transmitter.

noted as $\mathbf{r}(k, j)$ and can be written in vector form as

$$
\begin{aligned}
& \mathbf{r}(k, j)=\mathbf{A}(k, j) \mathbf{h}(j)+\mathbf{n}(k, j) \\
& =\mathbf{X}_{k}(j) \mathbf{h}_{k}(j)+\operatorname{ISI}(\mathrm{k}, \mathrm{j})+\operatorname{MAI}(\mathrm{k}, \mathrm{j})+\mathbf{n}(\mathrm{k}, \mathrm{j}) \in \mathbb{C}^{\mathrm{N}_{\mathrm{k}}}
\end{aligned}
$$

where the columns of the matrix $\mathbf{A}(k, j)$ are delayed versions of the transmitted chip sequences $\mathbf{a}_{k}(j)$ for $k=$ $1,2, \cdots, K$, one column per path. The length of the processing window $N_{k}$, is larger than the symbol interval $N$ to account for the asynchronous and multipath nature of the channel. The columns are weighted together by $\mathbf{h}(j)$, whose elements are the path gains of all users' paths. The received vector $\mathbf{r}(k, j)$ can be written as the sum of four terms: the signal of interest $\mathbf{X}_{k}(j) \mathbf{h}_{k}(j)$, the intersymbol interference (ISI), the multiple access interference (MAI), and the noise represented by $\mathbf{n}(k, j)$, which is a vector of complex Gaussian noise samples with zero mean and variance $N_{0}$. The columns of the matrix $\mathbf{X}_{k}(j)$ are essentially shifted versions of the chips due to the $k^{t h}$ user's $j^{t h}$ symbol, one column per path (the shift is determined by the path delay). The vector

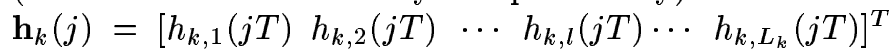
corresponds to the channel gains of the $k^{t h}$ user's paths, it is a part of $\mathbf{h}(j)$.

The task of the receiver is to detect the information bits from all users, i.e., detect $i_{k}(j)$ for $k=1,2, \ldots, K$ given the observation $\mathbf{r}(k, j)$. The decision on the $k^{t h}$ user's $j^{t h}$ symbol, is found as

$$
\hat{i}_{k}(j)=\arg \max _{m \in\{1,2, \cdots, M\}} z_{k}(m)
$$

where $z_{k}(m)$ is the decision statistic from symbol matched filter (MF) or multiuser detector (interference canceler in our case), based on the condition that the $m^{\text {th }}$ Walsh symbol is transmitted from user $k$.

With conventional MF, the soft decision is formed by correlating the received signal with the $M$ possible transmitted waveforms

$$
z_{k}(m)=\sum_{l=1}^{L_{k}}\left|\mathbf{x}_{k, l, m}^{*}(j) \mathbf{r}(k, j)\right|^{2}
$$

where $\mathbf{x}_{k, l, m}$ denotes the transmitted chip sequence due to the $k^{t h}$ user's $j^{t h}$ symbol from the $l^{t h}$ path based on the hypothesis that the $m^{t h}$ Walsh symbol is transmitted. It is formed by $\mathbf{s}_{m}$ scrambled with $\mathbf{C}_{k}(j)$ and compensated with the path delay $\tau_{k, l}$.

This simple scheme is particularly useful in the beginning of the detection process when the estimates of the fading channel are lacking, we must therefore carry out the detection in a noncoherent manner. However, it has poor performance in multiuser environment since it considers multiple access interference (MAI) as additive noise and the knowledge about MAI is not exploited in any way. An effective tool to increase the capacity of interference-limited CDMA systems is multiuser detection (MUD), a method of jointly detecting all the users in the system. Among different MUD techniques, the multistage interference cancellation schemes are known to be simple and effective for mitigation of MAI in long-code DS-CDMA systems. For the purpose of this study, we consider the PIC scheme introduced in [7].

The basic principle is that once the transmitted signals are estimated for all the users at the previous iteration, interference can be removed by subtracting the estimated signals of the interfering users from the received signal $\mathbf{r}(k, j)$ to form a new signal vector $\mathbf{r}^{\prime}(k, j)$ for demodulating the signal transmitted from user $k$, i.e.,

$$
\mathbf{r}^{\prime}(k, j)=\mathbf{r}(k, j)-\hat{\mathbf{A}}(k, j) \hat{\mathbf{h}}(j)+\hat{\mathbf{X}}_{k}(j) \hat{\mathbf{h}}_{k}(j)
$$

where $\mathbf{r}^{\prime}(k, j) \in \mathbb{C}^{N_{k}}$ denotes the interference canceled version of $\mathbf{r}(k, j)$ after subtracting the contributions from all the other users using decision feedback at the previous stage. The vector $\hat{\mathbf{A}}(k, j) \hat{\mathbf{h}}(j)$ represents the estimated contribution from all the users calculated by using the estimated data matrix $\hat{\mathbf{A}}(k, j)$ and channel vector $\hat{\mathbf{h}}(j)$. The vector $\hat{\mathbf{X}}_{k}(j) \hat{\mathbf{h}}_{k}(j)$ is the estimated contribution from all paths of user $k$. The soft decision with PIC is formed as

$$
z_{k}(m)=\operatorname{Re}\left\{\hat{\mathbf{h}}_{k}^{*}(j) \mathbf{X}_{k, m}^{*} \mathbf{r}^{\prime}(k, j)\right\}
$$

where $\mathbf{X}_{k, m}=\left[\begin{array}{llll}\mathbf{x}_{k, 1, m} & \mathbf{x}_{k, 2, m} & \cdots & \mathbf{x}_{k, L_{k}, m}\end{array}\right]$.

\section{TheORETICAL ANALYSIS}

\section{A. Performance analysis for non-coherent first stage}

To evaluate the probability of error, without loss of generality, let us assume that the $j^{\text {th }}$ symbol transmitted from the $k^{t h}$ user is the first Walsh symbol and the channel gain remains constant during one symbol interval. The decision 
statistic expressed in (2) can be reformed as

$$
z_{k}(m)= \begin{cases}\sum_{l=1}^{L_{k}}\left|d_{k, l}+m_{k, l}+i_{k, l}+n_{k, l}\right|^{2}, & \text { if } m=1 \\ \sum_{l=1}^{L_{k}}\left|m_{k, l}+i_{k, l}+n_{k, l}\right|^{2}, & \text { if } m \neq 1\end{cases}
$$

where $d_{k, l}=N h_{k, l}, m_{k, l}, i_{k, l}$, and $n_{k, l}$ stand for the desired signal, contribution from MAI, ISI, and noise, respectively.

In [8], the long pseudonoise sequences were modeled as a random binary sequence which led to the strong justification of an accurate Gaussian approximation based on the central limit theorem. This is the approach we take here to compute the variance of $m_{k, l}$ and $i_{k, l}$. If the processing gain is large enough, both MAI and ISI terms can be modeled as independent zero mean complex Gaussian random vectors and they are uncorrelated with the noise vector. Therefore, for the $k^{t h}$ user's $l^{\text {th }}$ receiver branch, the interference plus noise variance is $\sigma^{2}=\sigma_{m}^{2}+\sigma_{i}^{2}+\sigma_{n}^{2}$, where $\sigma_{m}^{2}=\operatorname{var}\left[m_{k, l}\right]=\mathrm{E}\left[\left|m_{k, l}\right|^{2}\right], \quad \sigma_{i}^{2}=\operatorname{var}\left[i_{k, l}\right]=$ $\mathrm{E}\left[\left|i_{k, l}\right|^{2}\right], \sigma_{n}^{2}=\operatorname{var}\left[n_{k, l}\right]=\mathrm{E}\left[\left|n_{k, l}\right|^{2}\right]$. Note that $\sigma^{2}, \sigma_{m}^{2}, \sigma_{i}^{2}$ depend on $k$ and $l$, which is not explicitly indicated in order to simplify notations. The noise variance can be easily computed as $\sigma_{n}^{2}=\sum_{n=1}^{n=N} N_{0}=N N_{0}$. According to [8], for direct-sequence systems with long spreading sequences, the elements (chips) of each ISI or MAI sequence corresponding to the $m^{t h}$ user's $i^{t h}$ path, can be approximated as statistically independent and each element can be treated as a zero mean Gaussian random variable with variance $\frac{2}{3} \mathrm{E}\left[\left|h_{m, i}\right|^{2}\right]=\frac{2}{3} P_{m, i}$ for chip asynchronous systems ${ }^{1}$ and $\stackrel{\mathrm{E}}{[}\left[\left|h_{m, i}\right|^{2}\right]=P_{m, i}$ for chip synchronous systems given $\psi(t)$ is a rectangular pulse. Due to space limit, we only derive the BER expressions for chip synchronous systems, and assume chip synchronism throughout this paper. However, the performance of chip asynchronous systems can be analyzed similarly. From the above analysis, we can derive the variance of the MAI and ISI as

$$
\begin{aligned}
\sigma_{i}^{2} & =\sum_{\substack{i=1 \\
i \neq l}}^{L_{k}} \sum_{n=1}^{N} \mathrm{E}\left[\left\|h_{k, i}\right\|^{2}\right]=N \sum_{\substack{i=1 \\
i \neq l}}^{L_{k}} P_{k, i} \\
\sigma_{m}^{2} & =\sum_{\substack{m=1 \\
m \neq k}}^{K} \sum_{i=1}^{L_{m}} \sum_{n=1}^{N} \mathrm{E}\left[\left\|h_{m, i}\right\|^{2}\right]=N \sum_{\substack{m=1 \\
m \neq k}}^{K} \sum_{i=1}^{L_{m}} P_{m, i}
\end{aligned}
$$

In case of equal gain among different diversity branches, i.e., $P_{k, 1}=P_{k, 2}=\cdots=P_{k, L_{k}}=P$, the interference variance does not differ from path to path. For the first stage noncoherent reception expressed in (4), the probability density function (pdf) of the decision statistics $z_{k}$ is given by

$$
f\left(z_{k}\right)= \begin{cases}\frac{1}{\sigma_{1}^{2 L_{k}}\left(L_{k}-1\right) !} z_{k}^{L_{k}-1} e^{\frac{-z_{k}}{\sigma_{1}^{2}}}, & \text { if } m=1 \\ \frac{1}{\sigma_{2}^{2 L_{k}}\left(L_{k}-1\right) !} z_{k}^{L_{k}-1} e^{\frac{-z_{k}}{\sigma_{2}^{2}}}, & \text { if } m \neq 1\end{cases}
$$

\footnotetext{
${ }^{1}$ For chip asynchronous system, time delays are assumed to be uniformed distributed over $\left[0, T_{c}\right]$, and $T_{c}$ is the chip interval.
}

which is central chi-square distribution with $2 L_{k}$ degrees of freedom. The variances $\sigma_{1}^{2}$ and $\sigma_{2}^{2}$ are computed as

$$
\begin{aligned}
\sigma_{1}^{2} & =\mathrm{E}\left(\left|N h_{k, l}+m_{k, l}+i_{k, l}+n_{k, l}\right|^{2}\right) \\
& =N^{2} P+\sigma_{m}^{2}+\sigma_{i}^{2}+\sigma_{n}^{2} \\
\sigma_{2}^{2} & =\sigma_{m}^{2}+\sigma_{i}^{2}+\sigma_{n}^{2}
\end{aligned}
$$

The probability of making correct symbol decision for user $k$ is calculated according to [9, p. 789] as

$$
\begin{aligned}
\mathcal{P}_{\mathrm{c}, k}= & \int_{0}^{\infty}\left[1-e^{-z_{k}} \sum_{l=0}^{L_{k}-1} \frac{z_{k}^{l}}{l !}\right]^{M-1} \frac{z_{k}^{L-1}}{(1+\gamma)^{L}\left(L_{k}-1\right) !} \\
& \cdot \exp \left(-\frac{z_{k}}{1+\gamma}\right) d z_{k}
\end{aligned}
$$

and the bit error probability is

$$
\mathcal{P}_{\mathrm{b}, k}=\frac{M}{2(M-1)}\left(1-\mathcal{P}_{\mathrm{c}, k}\right)
$$

where $\gamma=N^{2} P / \sigma_{2}^{2}$ is the average signal to interference plus noise ratio (SINR) per diversity branch.

\section{B. Performance analysis for multistage PIC}

The performance of coherent combining for single-user M-ary orthogonal systems with space diversity was analyzed in [3]. Here we extend its application to the analysis of PIC schemes in multiuser environments.

Let us assume that the first Walsh symbol was transmitted from the $k^{t h}$ user. The decision statistic expressed in (3) can be reformed after $p$ stages of cancellations

$$
\begin{aligned}
& z_{k}^{(p)}(m)=\sum_{l=1}^{L_{k}} \operatorname{Re}\left\{\hat{h}_{k, l}^{*} \mathbf{x}_{k, l, m} \mathbf{r}^{\prime}\right\} \\
& = \begin{cases}\sum_{l=1}^{L_{k}} d_{k, l}+m_{k, l}^{(p)}+i_{k, l}^{(p)}+n_{k, l}=d+n_{1}^{(p)}, & \text { if } m=1 \\
\sum_{l=1}^{L_{k}} m_{k, l}^{(p)}+i_{k, l}^{(p)}+n_{k, l}=n_{m}^{(p)}, & \text { if } m \neq 1\end{cases}
\end{aligned}
$$

where $d=\sum_{l=1}^{L_{k}} d_{k, l}=N \sum_{l=1}^{L_{k}} h_{k, l} \hat{h}_{k, l}^{*}$ is the desired signal. Assume accurate channel estimation, i.e., $\hat{h}_{k, l} \approx h_{k, l}$, then $d=\sum_{l=1}^{l=L_{k}} d_{k, l} \approx N \sum_{l=1}^{l=L_{k}}\left|h_{k, l}\right|^{2}$. The noise component for the $l^{t h}$ diversity branch is denoted by $n_{k, l}$. The contributions from MAI and ISI for the $l^{\text {th }}$ diversity branch at the $p^{t h}$ stage are denoted by $m_{k, l}^{(p)}$ and $i_{k, l}^{(p)}$ respectively. Using the Gaussian approximation, $n_{1}, n_{2}, \ldots, n_{M}$ are zero-mean statistically independent Gaussian random variables with equal variance $\left(\sigma^{2}\right)^{(p)} / 2$. The factor of $1 / 2$ is due to the fact that the $\operatorname{Re}(\cdot)$ operation in equation (7) removes the noise and interference present in the imaginary part of the decision statistics.

Let us denote $\mathcal{P}_{\mathrm{c}, k}^{(p)}(x)$ as the probability that the receiver makes correct symbol decision for user $k$ at the $p^{t h}$ stage conditioned on $x$, which is defined as $x=\frac{d}{\sigma^{(p)}}$. It is the probability that $z_{k}^{(p)}(1)=d+n_{1}^{(p)}$ is larger than 
each of the other $M-1$ outputs $z_{k}^{(p)}(2)=n_{2}^{(p)}, z_{k}^{(p)}(3)=$ $n_{3}^{(p)}, \ldots, z_{k}^{(p)}(M)=n_{M}^{(p)}[9]$ :

$\mathcal{P}_{\mathrm{c}, k}^{(p)}(x)=\frac{1}{\sqrt{2 \pi}} \int_{-\infty}^{\infty}[1-Q(y)]^{M-1} \exp \left[-\frac{1}{2}(y-\sqrt{2} x)^{2}\right] d y$

where the function $Q(x)$ is defined as $Q(x)=\frac{1}{2} \operatorname{erfc}\left(\frac{x}{\sqrt{2}}\right)$. The interference plus noise variance at the $p^{t h}$ stage is defined as $\left(\sigma^{2}\right)^{(p)}=\left(\sigma_{m}^{2}\right)^{(p)}+\left(\sigma_{i}^{2}\right)^{(p)}+\sigma_{n}^{2}$. The noise term does not change between iterations and can be computed as $\sigma_{n}^{2}=N N_{0} \sum_{l=1}^{L_{k}}\left|h_{k, l}\right|^{2}$.

In the derivation of the variance of MAI, which changes at each iteration due to interference cancellation, we utilize some distinct feature of the Walsh code as depicted by Table I and II. The new vector $\mathbf{r}^{\prime}$ is obtained by canceling other user's distribution path-by-path using the decision feedback from the $(p-1)^{t h}$ stage. At the $p^{t h}$ stage, the probability of correct cancellation is $\mathcal{P}_{\mathrm{c}, m}^{(p-1)}=1-\mathcal{P}_{\mathrm{e}, m}^{(p-1)}$, where the interfering user $m=1, \ldots, K$, and $m \neq k$. The variance of the remaining MAI after correct cancellation (or cancellation residual) is of course zero. On the other hand, in case of erroneous cancellation, which occurs with probability $\mathcal{P}_{\mathrm{e}, m}^{(p-1)}$, the cancellation residual is determined by the difference of two distinct Walsh symbols. Table II indicates that if a Walsh codeword is subtracted by another Walsh codeword, the resulting word $\triangle \mathbf{w}$ contains $\frac{M}{2}$ number of zeros and $\frac{M}{2}$ number of $\pm 2 \mathrm{~s}$. Although Table II is not exhaustive, the rest of the words can be easily computed from Table I and shown to comply with the same rule. We use $M=8$ as an example in these tables; however, the conclusion applies to any value of $M$. The cancellation residual for each path is formed by spreading $\Delta \mathbf{w}$ to a number of $N$ chips (which consequently contains $\frac{N}{2}$ number of zeros and $\frac{N}{2}$ number of $\pm 2 \mathrm{~s}$ ), scrambling with a random code, then multiplying the scrambled sequence with channel coefficient $h_{m, i}$, where $i=1,2, \ldots, L_{m}$. The variance of MAI in the $l^{t h}$ diversity branch after cancellation is therefore

$$
\begin{aligned}
&\left(\sigma_{m}^{2}\right)_{l}^{(p)}=\left(1-\mathcal{P}_{\mathrm{e}, m}^{(p-1)}\right) \cdot 0+\left|h_{k, l}\right|^{2}( \pm 2)^{2} \frac{N}{2} \\
& \cdot \sum_{\substack{m=1 \\
m \neq k}}^{K} \sum_{i=1}^{L_{m}} \mathcal{P}_{\mathrm{e}, m}^{(p-1)} \mathrm{E}\left[\left|h_{m, i}\right|^{2}\right] \\
&=\left|h_{k, l}\right|^{2} 2 N \sum_{\substack{m=1 \\
m \neq k}}^{K} \sum_{i=1}^{L_{m}} \mathcal{P}_{\mathrm{e}, m}^{(p-1)} P_{m, i}
\end{aligned}
$$

The variance of MAI from all the diversity branches of user $k$ can therefore be computed as

$$
\left(\sigma_{m}^{2}\right)^{(p)}=\sum_{l=1}^{L_{k}}\left(\sigma_{m}^{2}\right)_{l}^{(p)}=\sum_{l=1}^{L_{k}}\left|h_{k, l}\right|^{2} 2 N \sum_{\substack{m=1 \\ m \neq k}}^{K} \sum_{i=1}^{L_{m}} \mathcal{P}_{\mathrm{e}, m}^{(p-1)} P_{m, i}
$$

Next, we derive the variance of the self interference for user $k$. Denote $p_{k, i}$ and $p_{k, l}$ as integer part of the delays
TABLE I

MAPPING BETWEEN INPUT BITS AND WALSH CODEWORDS

\begin{tabular}{|c|c|c|c|}
\hline $\mathbf{w}_{0}-\mathbf{w}_{1}$ & 000002222 & & \\
\hline $\mathbf{w}_{0}-\mathbf{w}_{2}$ & 00220022 & $\mathbf{w}_{1}-\mathbf{w}_{2}$ & \\
\hline $\mathbf{w}_{0}-\mathbf{w}_{3}$ & 00222200 & $\mathbf{w}_{1}-\mathbf{w}_{3}$ & $\begin{array}{llllllll}0 & 0 & 2 & 2 & 0 & 0 & -2 & -2\end{array}$ \\
\hline $\mathbf{w}_{0}-\mathbf{w}_{4}$ & 020020202 & $\mathbf{w}_{1}-\mathbf{w}_{4}$ & $\begin{array}{llllllll}0 & 2 & 0 & 2 & -2 & 0 & -2 & 0\end{array}$ \\
\hline $\mathbf{w}_{0}-\mathbf{w}_{5}$ & 02022020 & $\mathbf{w}_{1}-\mathbf{w}_{5}$ & $\begin{array}{llllllll}0 & 2 & 0 & 2 & 0 & -2 & 0 & -2\end{array}$ \\
\hline $\mathbf{w}_{0}-\mathbf{w}_{6}$ & 02200220 & $\mathbf{w}_{1}-\mathbf{w}_{6}$ & $\begin{array}{llllllll}0 & 2 & 2 & 0 & -2 & 0 & 0 & -2\end{array}$ \\
\hline $\mathbf{w}_{0}-\mathbf{w}_{7}$ & 02202002 & $\mathbf{w}_{1}-\mathbf{w}_{7}$ & $\begin{array}{lllllllll}0 & 2 & 2 & 0 & 0 & -2 & -2 & 0\end{array}$ \\
\hline
\end{tabular}

\begin{tabular}{|c|c|c|}
\hline \hline info bits & index & Walsh codeword \\
\hline & $m=i_{k}(j)$ & $\mathbf{w}_{m}$ \\
\hline$+1+1+1$ & 0 & $\mathbf{w}_{0}:+1+1+1+1+1+1+1+1$ \\
\hline$+1+1-1$ & 1 & $\mathbf{w}_{1}:+1+1+1+1-1-1-1-1$ \\
\hline$+1-1+1$ & 2 & $\mathbf{w}_{2}:+1+1-1-1+1+1-1-1$ \\
\hline$+1-1-1$ & 3 & $\mathbf{w}_{3}:+1+1-1-1-1-1+1+1$ \\
\hline$-1+1+1$ & 4 & $\mathbf{w}_{4}:+1-1+1-1+1-1+1-1$ \\
\hline$-1+1-1$ & 5 & $\mathbf{w}_{5}:+1-1+1-1-1+1-1+1$ \\
\hline$-1-1+1$ & 6 & $\mathbf{w}_{6}:+1-1-1+1+1-1-1+1$ \\
\hline$-1-1-1$ & 7 & $\mathbf{w}_{7}:+1-1-1+1-1+1+1-1$ \\
\hline
\end{tabular}

TABLE II

DifFERENCE BETWEEN DIFFERENT WALSh CODEWORDS

$\tau_{k, i}$ and $\tau_{k, l}$. For the $l^{\text {th }}$ diversity branch, the $i^{\text {th }}$ ISI vector $\left(i=1, \ldots, L_{k}, i \neq l\right)$ due to the $k^{t h}$ user's $j^{t h}$ symbol (the desired symbol) spans $N-\left|p_{k, i}-p_{k, l}\right|$ chips. Its variance is computed as $\left|h_{k, l}\right|^{2} \cdot\left(N-\left|p_{k, i}-p_{k, l}\right|\right) P_{k, i}$, for the same reasoning as stated in Section III-A, and it does not change at each iteration. The ISI component due to some other symbol spans $\left|p_{k, i}-p_{k, l}\right|$ chips, it is canceled with decision feedback at each iteration. It can be treated in the same way as MAI, its variance is therefore $\left|h_{k, l}\right|^{2} \cdot 2\left|p_{k, i}-p_{k, l}\right| \mathcal{P}_{\mathrm{e}, k}^{(p-1)} P_{k, i}$. The variance the total ISI term can therefore be computed as

$$
\left(\sigma_{i}^{2}\right)^{p}=\sum_{l=1}^{L_{k}}\left|h_{k, l}\right|^{2} \sum_{\substack{i=1 \\ i \neq l}}^{L_{k}}\left[N+\left(2 \mathcal{P}_{\mathrm{e}, k}^{(p-1)}-1\right)\left|p_{k, i}-p_{k, l}\right|\right] P_{k, i}
$$

Based on the above analysis, we derive the total noise plus interference variance as $\left(\sigma^{2}\right)^{(p)}=\sigma_{n}^{2}+\left(\sigma_{i}^{2}\right)^{(p)}+$ $\left(\sigma_{m}^{2}\right)^{(p)}=\sum_{l=1}^{L_{k}}\left|h_{k, l}\right|^{2}\left(\alpha_{l}^{2}\right)^{(p)}$, and $\left(\alpha_{l}^{2}\right)^{(p)}$ is defined as

$$
\begin{aligned}
\left(\alpha_{l}^{2}\right)^{(p)}=N N_{0}+2 N \sum_{\substack{m=1 \\
m \neq k}}^{K} \sum_{i=1}^{L_{m}} \mathcal{P}_{\mathrm{e}, m}^{(p-1)} P_{m, i} \\
+\sum_{\substack{i=1 \\
i \neq l}}^{L_{k}}\left[N+\left(2 \mathcal{P}_{\mathrm{e}, k}^{(p-1)}-1\right)\left|p_{k, i}-p_{k, l}\right|\right] P_{k, i}
\end{aligned}
$$

In case of equal power among different paths, i.e., $P_{k, 1}=$ $P_{k, 2}=\cdots=P_{k, L_{k}}=P$, then $\left(\alpha_{1}^{2}\right)^{(p)}=\left(\alpha_{2}^{2}\right)^{(p)}=\cdots=$ $\left(\alpha_{L_{k}}^{2}\right)^{(p)}=\left(\alpha^{2}\right)^{(p)}$. Denote

$$
\begin{aligned}
& x=\frac{d}{\sigma^{(p)}}=\frac{N \sum_{l=1}^{L_{k}}\left|h_{k, l}\right|^{2}}{\alpha^{(p)} \sqrt{\sum_{l=1}^{L_{k}}\left|h_{k, l}\right|^{2}}}=\frac{N}{\alpha^{(p)}} \sqrt{\sum_{l=1}^{L_{k}}\left|h_{k, l}\right|^{2}} \\
& z=x^{2}=\frac{N^{2}}{\left(\alpha^{2}\right)^{(p)}} \sum_{l=1}^{l=L_{k}}\left|h_{k, l}\right|^{2}
\end{aligned}
$$


The random variable $z$ is central chi-square distributed with $2 L_{k}$ degrees of freedom and probability density function

$$
p(z)=\frac{z^{L_{k}-1} \exp \left(-z / \gamma^{(p)}\right)}{\gamma^{(p) L_{k}}\left(L_{k}-1\right) !} ; 0 \leq z \leq \infty
$$

where $\gamma^{(p)}=\frac{N^{2}}{\left(\alpha^{2}\right)^{(p)}} \mathrm{E}\left[\left|h_{k, l}\right|^{2}\right]=\frac{N^{2} P}{\left(\alpha^{2}\right)^{(p)}}$ stands for the average SINR of each diversity branch. Consequently,

$$
p(x)=\frac{2 x^{2 L_{k}-1} \exp \left(-x^{2} / \gamma^{(p)}\right)}{\gamma^{(p) L_{k}}\left(L_{k}-1\right) !} ; 0 \leq x \leq \infty
$$

To obtain the error probability when $x$ is random, we must average $\mathcal{P}_{\mathrm{c}, k}^{(p)}(x)$ given in (8) over the distribution of $x$, i.e.,

$$
\mathcal{P}_{\mathrm{c}, k}^{(p)}=\mathrm{E}\left[\mathcal{P}_{\mathrm{c}, k}^{(p)}(x)\right]=\int_{0}^{\infty} \mathcal{P}_{\mathrm{c}, k}^{(p)}(x) p(x) d x
$$

Without carrying out the integration, $\mathcal{P}_{\mathrm{c}, k}^{(p)}$ can be approximated according to [10] by

$\mathcal{P}_{\mathrm{c}, k}^{(p)} \approx \frac{2}{3} \mathcal{P}_{\mathrm{c}, k}^{(p)}\left(\mu_{x}\right)+\frac{1}{6} \mathcal{P}_{\mathrm{c}, k}^{(p)}\left(\mu_{x}+\sqrt{3} \sigma_{x}\right)+\frac{1}{6} \mathcal{P}_{\mathrm{c}, k}^{(p)}\left(\mu_{x}-\sqrt{3} \sigma_{x}\right)$

where $\mu_{x}$ and $\sigma_{x}$ are the mean and standard deviation of $x$

$$
\begin{aligned}
\mu_{x} & =\mathrm{E}[x]=\int_{0}^{\infty} x p(x) d x \\
& =\frac{2}{\gamma^{(p) L_{k}}\left(L_{k}-1\right) !} \int_{0}^{\infty} x^{2 L_{k}} \exp \left(-x^{2} / \gamma^{(p)}\right) d x \\
\mathrm{E}\left[x^{2}\right] & =\frac{N^{2}}{\left(\alpha^{2}\right)^{(p)}} \sum_{l=1}^{L_{k}} \mathrm{E}\left[\left|h_{k, l}\right|^{2}\right]=\frac{N^{2} L_{k} P}{\left(\alpha^{2}\right)^{(p)}} \\
\sigma_{x} & =\sqrt{\mathrm{E}\left[x^{2}\right]-\mu_{x}^{2}}
\end{aligned}
$$

With $\mathcal{P}_{c, k}^{(p)}$ calculated in (10), the bit error probability $\mathcal{P}_{\mathrm{b}, k}$ at the $p^{t h}$ cancellation stage can be derived as

$$
\mathcal{P}_{\mathrm{b}, k}^{(p)}=\frac{M}{2(M-1)} \mathcal{P}_{\mathrm{e}, k}^{(p)}=\frac{M}{2(M-1)}\left(1-\mathcal{P}_{\mathrm{c}, k}^{(p)}\right)
$$

and initialized with $\mathcal{P}_{\mathrm{b}, k}^{(1)}$ which is computed according to (5) and (6).

\section{Analytical Results and Performance COMPARISON}

Comparison between analysis and simulation is presented in this section. In our simulations, each user transmits one of $M=8$ Walsh codes spread to a total length of $N=64$ chips. The effective spreading of the system is $N / \log _{2} M=64 / 3$ chips per bit. Different users are separated by different scrambling codes $\mathbf{C}_{k}(j)$ which are random, and differ from symbol to symbol. Channels are independent Rayleigh fading channels. The normalized
Doppler frequency is assumed to be $f_{d} T=0.01$. The number of multipath channels $L_{k}$ is set to be $4,\left(L_{k}=L=4\right)$ for all $k$. Perfect slow power control is assumed in the sense that $P_{k}=\sum_{l=1}^{L_{k}} P_{k, l}$, the average received power, is equal for all users. Different paths are assumed to have equal gain and the channel coefficients are normalized so that each user has unity gain, i.e., $P_{k, 1}=P_{k, 2}=\cdots=P_{k, L_{k}}$ and $P_{k}=\sum_{l=1}^{L_{k}} P_{k, l}=1$. For simplicity, the simulated system is assumed to be chip-synchronous, i.e., all path delays are assumed to be multiples of $T_{c}$. However, the system is asynchronous on the symbol level. The simulation results are averaged over random distributions of fading, noise, delay, and scrambling code through numerous Monte-Carlo runs.

Noncoherent equal gain combining is used for the first stage of the PIC scheme to account for the fact that channel estimates are not yet available at the initial iteration. In the following stages, both interference cancellation and channel estimation are carried out in decision directed mode using the detected data from the previous iteration. Channel estimation is conducted with the Maximum Likelihood algorithm introduced in [7].

Figures $2-4$ shows the comparison between analytical and simulated results for different number of users. The simulated curves precisely match the theoretical ones for the first noncoherent stage, which proves that Gaussian approximation is accurate to model MAI and ISI sequences as well as the elements of each interference sequence in long-code systems. The analysis starts to deviate slightly from simulations, but is still fairly accurate, after the first noncoherent stage. The theoretical analysis is a little pessimistic when the system is too lightly loaded, and a little optimistic when the system is too heavily loaded. From both simulation and analysis, one can observe that it takes PIC more stages to converge as $K$ increases (the system becomes more heavily loaded). Seven stages (excluding the first noncoherent stage) ought be enough for the system to reach convergence in any case.

System capacity is illustrated in Figure 5 by plotting BER as a function of the number of users using both analytical and simulated results. It is clearly shown that analysis is in close agreement with simulation for BER above $10^{-4}$. However, the analysis tends to over-estimate the MAI when the number of users is very small. Conversely, the MAI is under-estimated when there are too many active users. Compared with the topmost curve which represents the first noncoherent stage, the subsequent PIC stages significantly increase system capacity and BER performance as indicated by both analysis and simulation.

\section{Conclusions}

BER performance of the multistage PIC scheme is theoretically analyzed in this paper for the orthogonally modulated long-code CDMA system under frequency selective Rayleigh fading channels. Comparison with the simulated results shows that the analysis is fairly accurate. A close agreement is seen between analysis and simulation in most cases except for low BER (below $10^{-4}$ ). The analysis tends 


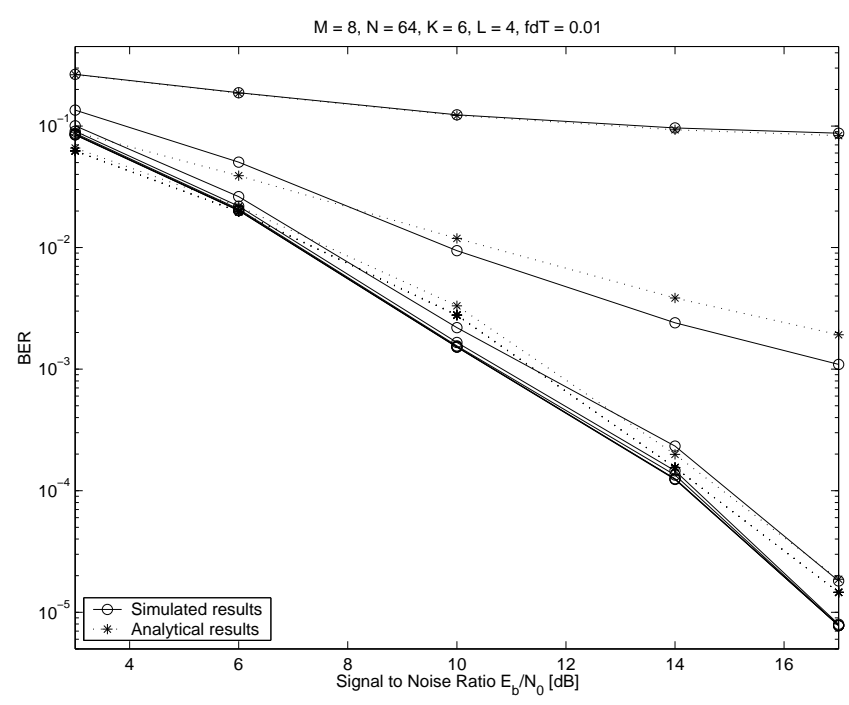

Fig. 2. Analysis vs. simulation, $K=6$.

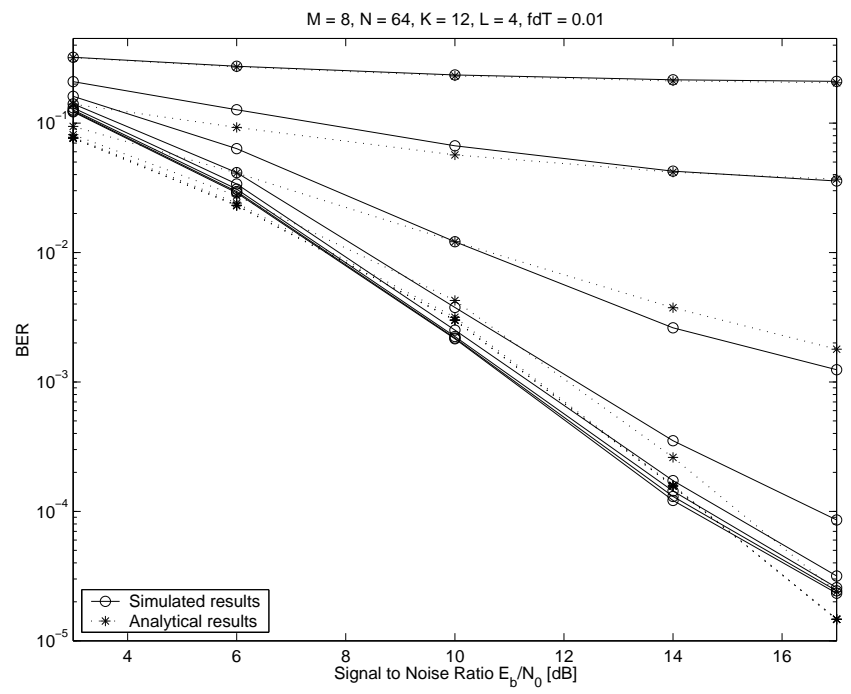

Fig. 3. Analysis vs. simulation, $K=12$.

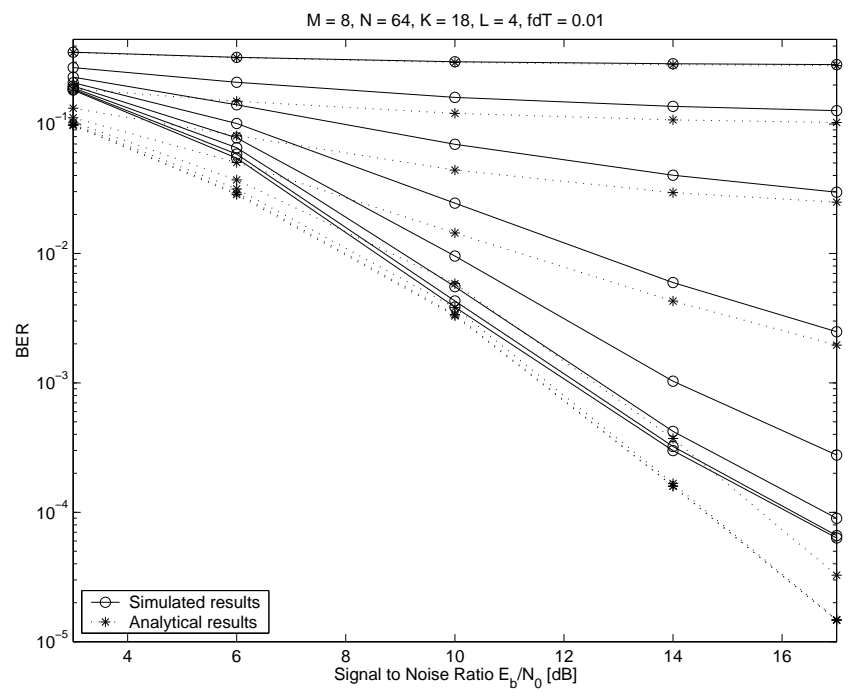

Fig. 4. Analysis vs. simulation, $K=18$.

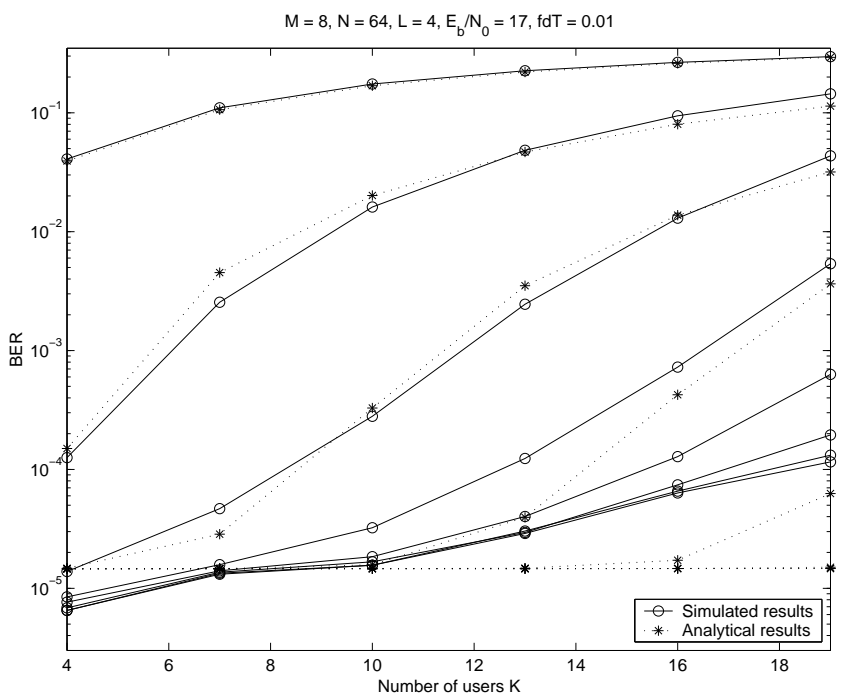

Fig. 5. Analysis vs. simulation, system capacity with 7-stage PIC.

to over estimate MAI in very lightly loaded systems, and under estimate MAI in very heavily loaded system. Considering the fact that the target BER for an uncoded system is usually above $10^{-4}$, our analytical results are quite satisfactory. The presented analytical method provides an effective measure to predict BER performance and system capacity for the PIC scheme under investigation.

\section{REFERENCES}

[1] A. Naguib, A. Paulraj. "Performance of Wireless CDMA with M-ary orthogonal modulation and cell site antenna arrays". IEEE Journal on Selected Areas Communications, vol. 14, no. 9, pp. 1770-1783, Dec. 1996.

[2] L. Jalloul, J. Holtzman. "Performance analysis of DS/CDMA with noncoherent $\mathrm{M}$-ary orthogonal modulation in multipath fading channels". IEEE Journal on Selected Areas in Communications, vol. 12, no. 5, pp. 862-870, June 1994.

[3] J. Thompson, P. Grant, B. Mulgrew. "Algorithms for coherent diversity combining of M-ary orthogonal signals". IEEE Journal on Selected Areas in Communications, vol. 17, no. 11, pp. 18861899, Nov. 1999.

[4] A. Kaul, B. Woerner. "Analytic limits on performance of adaptive multistage interference cancellation for CDMA". Electronics Letters, vol. 30, no. 25, pp. 2093-2095, Dec. 1994.

[5] R. Buehrer, B. Woerner. "Analysis of adaptive multistage interference cancellation for CDMA using an improved Gaussian approximation". IEEE Transactions on Communications, vol. 44, no. 10, pp. 1308-1321, Oct. 1996.

[6] G. Xue, J. Weng, T. Le-Ngoc, S. Tahar. "An analytical model for performance evaluation of parallel interference canceller in CDMA systems". IEEE Communications Letters, vol. 4, no. 6, pp. 184-186, June 2000.

[7] E. Ström, S. Miller. "Iterative demodulation and channel estimation of orthogonal signalling formats in asynchronous DS-CDMA systems". IEICE Transactions on Electronics, vol. E85-C, no. 3, pp. 442-451, March 2002.

[8] D. Torrieri. "Performance of direct-sequence systems with long pseudonoise sequences". IEEE Journal on Selected Areas in Communications, vol. 10, no. 4, pp. 770-781, May 1992.

[9] J. Proakis. Digital Communications, 3rd edition, McGraw-Hill, 1995.

[10] J. Holtzman. "A simple, accurate method to calculate spreadspectrum multiple-access error probabilities". IEEE Transactions on Communications, vol. 40, no. 3, pp. 461-464, March 1992. 